\title{
Sustainability in intelligent building environments using weighted priority scheduling algorithm
}

\begin{abstract}
Intelligent buildings require various gadgets and sensors to automatically control the underlying environments with enhanced levels of comfort and efficiency. The ever-evolving technology and increasingly complex devices operated by heterogeneous systems have created challenges for intelligent building environments. Multi-platform type of heterogeneous systems functioning in intelligent building environments can lead to system failures with regard to deadlocks and unpredictable behavior, known as conflict occurrence. In this paper, we propose a new decision-making model with a weighted-priority scheduling algorithm that solves the conflicts to achieve efficient and sustainable communication response among heterogeneous systems. The experimental results demonstrate the enhanced response time of the model with conflict resolution. Furthermore, this model minimizes the external intervention to control the setting of such buildings.
\end{abstract}

Keyword: Conflict resolution; Machine learning; Priority scheduling; Intelligent buildings 